\title{
Studies on alpha-induced astrophysical reactions using the low- energy RI beam separator CRIB
}

H. Yamaguchi, ${ }^{1, a}$, D. Kahl ${ }^{1}$, T. Nakao ${ }^{1}$, Y. Wakabayashi ${ }^{2}$, S. Kubono ${ }^{2}$, T. Hashimoto ${ }^{3}$, S. Hayakawa $^{4}$, T. Kawabata ${ }^{5}$, N. Iwasa ${ }^{6}$, T. Teranishi ${ }^{7}$, Y.K. Kwon ${ }^{8}$, P.S. Lee ${ }^{9}$, D.N. Binh ${ }^{10}$, L.H. Khiem $^{10}$, and N.G. Duy ${ }^{10}$

${ }^{1}$ Center for Nuclear Study, the University of Tokyo, 2-1 Hirosawa, Wako, Saitama, Japan

${ }^{2}$ The Institute of Physical and Chemical Research (RIKEN), 2-1 Hirosawa, Wako, Saitama 351-0198, Japan

${ }^{3}$ Research Center for Nuclear Physics, Osaka University, 10-1 Mihogaoka, Ibaraki, Osaka 567-0047, Japan

${ }^{4}$ Laboratori Nazionali del Sud, Via S. Sofia 62, 95125 Catania, Italy

${ }^{5}$ Department of Physics, Kyoto University, Kita-Shirakawa, Kyoto 606-8502, Japan

${ }^{6}$ Department of Physics, Tohoku University, Aoba, Sendai, Miyagi 980-8578, Japan

${ }^{7}$ Department of Physics, Kyushu University, 6-10-1 Hakozaki, Fukuoka 812-8581, Japan

${ }^{8}$ Institute for Basic Science, 70, Yuseong-daero 1689-gil, Yuseong-gu, Daejeon 305-811, Republic of Korea

${ }^{9}$ Department of Physics, Chung-Ang University, Seoul 156-756, Republic of Korea

${ }^{10}$ Institute of Physics, Vietnam Academy of Science and Technology, 18 Hong Quoc Viet, Nghia do, Hanoi, Vietnam

\begin{abstract}
Several alpha-induced astrophysical reactions have been studied at CRIB (CNS Radioactive Ion Beam separator), which is a low-energy RI beam separator at Center for Nuclear Study (CNS) of the University of Tokyo. Two major methods to study them are the $\alpha$ resonant scattering, and direct measurements of $(\alpha, p)$ reactions using an active or inactive helium gas target. Among the recent studies at CRIB, the measurement of ${ }^{7} \mathrm{Be}+\alpha$ resonant scattering is discussed.
\end{abstract}

\section{Introduction}

CRIB [1, 2] is a radio-isotope (RI) beam separator operated by Center for Nuclear Study (CNS), the University of Tokyo, installed in the RIBF facility of RIKEN Nishina Center. CRIB can produce lowenergy $(<10 \mathrm{MeV} / \mathrm{u}) \mathrm{RI}$ beams in flight, using primary heavy-ion beams from the AVF cyclotron of RIKEN $(\mathrm{K}=70)$. Most of the RI beams are produced via direct reactions such as $(p, n),(d, p)$ and $\left({ }^{3} \mathrm{He}, n\right)$, taking place at an 8 -cm-long gas target with a maximum pressure of 760 Torr. A cryogenic target system, in which the target gas can be cooled down to about $90 \mathrm{~K}$, is currently available, and an intense ${ }^{7} \mathrm{Be}$ beam of $2 \times 10^{8}$ pps was produced using the system [3]. The secondary beam is purified with an magnetic analysis using dipole magnets, and with a Wien filter, which can separate the beams according to their velocities. For relatively light RI beams such as ${ }^{7} \mathrm{Be}$, we can obtain a good purity of almost $100 \%$ after the Wien filter.

The low-energy RI beams at CRIB are suitable for studies of astrophysical reactions and nuclear structure. An experimental method extensively used was the thick-target in inverse kinematics method

\footnotetext{
ae-mail: yamag@cns.s.u-tokyo.ac.jp
} 
[4]. In that method, the beam energy is degraded in a thick reaction target, and reactions occur at various center-of-mass energies. We detect light particles emitted after reactions, and reconstruct the kinematics. This method has several advantages, namely, (a) using inverse kinematics, we can study reactions with short-lived RI which cannot be used as the target, (b) we can perform simultaneous measurements of cross section of various excitation energies without varying the incoming RI beam energy, and (c) we can perform measurements at $180^{\circ}$ in center-of-mass angle, where the Coulomb scattering is minimal. Many fruitful results have been obtained at CRIB with this method [5-9].

A recent major topic of our interest is the measurement of alpha-induced reactions. Several $(\alpha, p)$ reactions, such as ${ }^{14} \mathrm{O}(\alpha, p),{ }^{11} \mathrm{C}(\alpha, p),{ }^{21} \mathrm{Na}(\alpha, p),{ }^{18} \mathrm{Ne}(\alpha, p)$, and ${ }^{30} \mathrm{~S}(\alpha, p)$ have been measured at CRIB. The elastic resonant scatterings of $\alpha$ particle using ${ }^{14} \mathrm{O},{ }^{21} \mathrm{Na},{ }^{7} \mathrm{Li}[9]$ and ${ }^{7} \mathrm{Be}$ beams have also been measured. These measurements are related to astrophysical $(\alpha, \gamma)$ reaction rates, and are also relevant for studying nuclear cluster structure. Another possible method to study $(\alpha, \gamma)$ reactions using $\mathrm{RI}$ beams is to measure the $\left({ }^{6} \mathrm{Li}, d\right)$ transfer reaction. This could be applied, for example, ${ }^{15} \mathrm{O}$ beam to study the ${ }^{15} \mathrm{O}(\alpha, \gamma)$ reaction. However, the beam intensity would be a key issue when we use RI beams. According to our estimation, an intense ${ }^{15} \mathrm{O}$ beam of $10^{8}$ pps-order is required to resolve the relevant resonance at $4.03 \mathrm{MeV}$ from others. Among the performed experiments, the one on ${ }^{7} \mathrm{Be}+\alpha$ scattering measurement, which is related to the cluster structure of ${ }^{11} \mathrm{C}$, is presented below.

\section{Beryllium-7+ $\alpha$ elastic resonant scattering}

The measurement of ${ }^{7} \mathrm{Be}+\alpha$ scattering can be associated with the ${ }^{7} \operatorname{Be}(\alpha, \gamma)$ reaction. The ${ }^{7} \operatorname{Be}(\alpha, \gamma)$ reaction is considered to play an important role in the hot $p$ - $p$ chain and related reaction sequences [10]. Several reaction sequences including the ${ }^{7} \operatorname{Be}(\alpha, \gamma)$ reaction should take place in some hightemperature environments at $T_{9}>0.2$, where $T_{9}$ is the temperature in GK. In the $v p$-process in core-collapse supernovae [11], the ${ }^{7} \operatorname{Be}(\alpha, \gamma)$ reaction may contribute as much as the triple- $\alpha$ process to the synthesis of elements heavier than boron at the relevant temperature of $T_{9}=1.5-3$, according to a theoretical calculation [12]. The Gamow energy window at the highest temperature $T_{9}=3$ corresponds to the excitation energy $E_{\mathrm{ex}}=8.2-9.6 \mathrm{MeV}$ in ${ }^{11} \mathrm{C}$. By our study, the resonant reaction rate should be evaluated more precisely by determining $\alpha$ widths for the resonances at high temperatures.

In 2010, we performed the measurement of ${ }^{7} \mathrm{Be}+\alpha$ resonant elastic and inelastic scatterings with the thick-target method in inverse kinematics at CRIB [13]. A low-energy ${ }^{7} \mathrm{Be}$ beam at $14.7 \mathrm{MeV}$ was produced using a $2.3-\mathrm{mg} / \mathrm{cm}^{2}$-thick hydrogen gas target and a primary ${ }^{7} \mathrm{Li}$ beam at $5.0 \mathrm{MeV} / \mathrm{u}$. The typical ${ }^{7}$ Be beam intensity used in the measurement was $1-2 \times 10^{5}$ per second at the secondary target, and the main measurement using a thick helium-gas target was performed for 4 days, injecting $2.9 \times 10^{10}{ }^{7} \mathrm{Be}$ particles into the target. We obtained an excitation function with several peaks, which correspond to the resonance structure in ${ }^{11} \mathrm{C}$. An R-matrix analysis was performed to deduce the parameters of these resonances, as illustrated in Fig. 1. A similar measurement was independently carried out at other facilities [14], but our measurement was with $\gamma$-ray detection to identify inelastic scatterings, and several differences are found in the obtained spectra [13].

\section{Astrophysical reaction rate of ${ }^{7} \mathrm{Be}(\alpha, \gamma)$}

The resonances observed in the present work might contribute to the astrophysical ${ }^{7} \mathrm{Be}(\alpha, \gamma){ }^{11} \mathrm{C}$ reaction rate at high temperature, $T_{9}>1.5$. Here we calculate the resonant reaction rate and compare it with the total reaction rate evaluated in NACRE $[15,16]$. In the evaluation in NACRE, only 2 resonances at 8.1045 and $8.420 \mathrm{MeV}$ are included. These two resonances dominate the reaction rate $N_{\mathrm{A}}\langle\sigma v\rangle$ up to the temperature $T_{9} \sim 3$, and a Hauser-Feshbach calculation was used to provide the 
reaction rate at higher temperatures. The resonant reaction rates were calculated for three resonances using analytical formula described in [15], and plotted in Fig. 1. Table 1 shows the parameters we
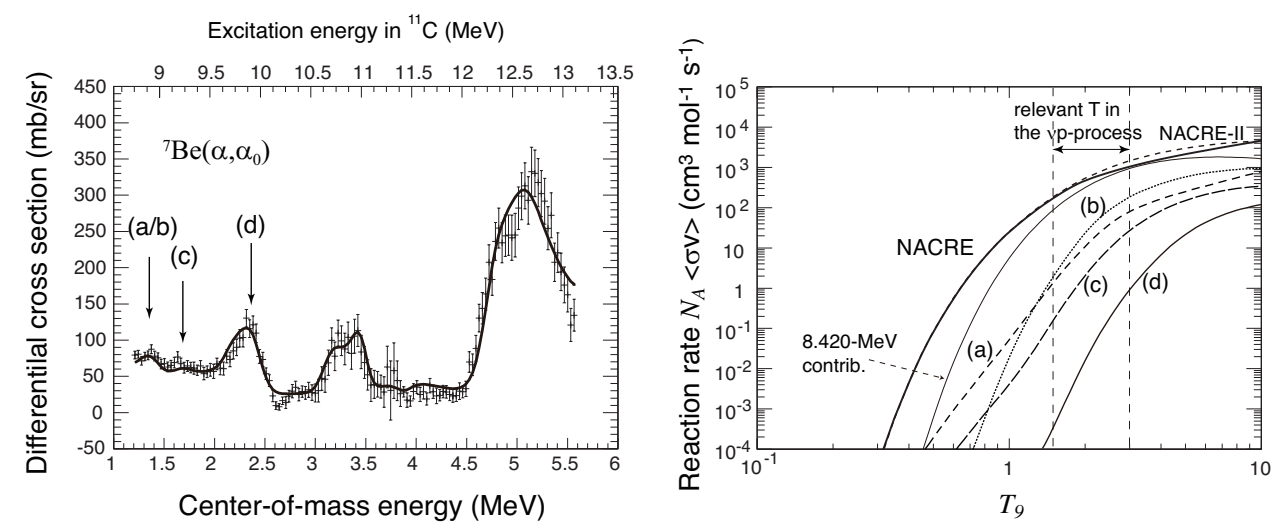

Figure 1. Excitation function of ${ }^{7} \mathrm{Be}+\alpha$ elastic scattering with an R-matrix fit curve (left panel) and resonant reaction rate of ${ }^{7} \mathrm{Be}(\alpha, \gamma)$ for the $8.90,9.20$, and $9.97-\mathrm{MeV}$ resonances, calculated by the analytical formula (right panel, see text and Table 1 for the labels). The evaluation by NACRE and NACRE-II are shown for comparison. The contribution by the $8.420-\mathrm{MeV}$ resonance, included in NACRE, is also shown.

Table 1. Parameters used in the reaction rate calculation. The dominant destination states of the $\gamma$ decay according to our calculation are also shown.

\begin{tabular}{ccccccccc}
\hline \hline & $E_{\mathrm{ex}}$ & $J^{\pi}$ & $\Gamma_{\text {tot }}(\mathrm{keV})$ & $\Gamma_{\alpha}(\mathrm{keV})$ & $\Gamma_{\gamma}(\mathrm{eV})$ & $\omega$ & $\omega \gamma(\mathrm{eV})$ & dominant dest. \\
\hline (a) & 8.90 & $9 / 2^{+}$ & 8 & 8 & 0.48 & 2.5 & 1.2 & $8.655 \mathrm{MeV}\left(7 / 2^{+}\right)$ \\
(b) & 8.90 & $3 / 2^{+}$ & 8 & 8 & 1.7 & 1.0 & 1.7 & ground $\left(3 / 2^{-}\right)$ \\
(c) & 9.20 & $5 / 2^{+}$ & 500 & 13 & 34 & 1.5 & 1.3 & ground $\left(3 / 2^{-}\right)$ \\
(d) & 9.97 & $7 / 2^{-}$ & 218 & 153 & 0.97 & 2.0 & 1.4 & $6.90 \mathrm{MeV}\left(5 / 2^{+}\right)$ \\
\hline \hline
\end{tabular}

used in the calculation. The $\Gamma_{\gamma}$ and the decay scheme are experimentally unknown for this energy range. Therefore, we evaluated $\Gamma_{\gamma}$ by a calculation based on the Weisskopf unit with a spectroscopic factor of 0.1 , which roughly reproduces the experimentally known $\Gamma_{\gamma}$ of the 8.1045 and $8.420-\mathrm{MeV}$ resonances. Note that such a spectroscopic factor was not explicitly used in the plot of our previous publication [9]. The labels (a)-(d) in Fig. 1 correspond to the ones in Table 1. (a) is for our newly identified resonance at $8.90 \mathrm{MeV}$. $J^{\pi}$ was taken to be $9 / 2^{+}$from our best fit. Since the $J^{\pi}$ assignment may not be correct, we also evaluated the contribution for the same resonance, but when the resonance had a lower spin of $3 / 2$, shown as (b). Basically (b) contributes to the reaction rate more, but its tail contribution is smaller than (a). For $T_{9}=2-3$, where the $8.420-\mathrm{MeV}$ resonance dominates the reaction rate, the contribution of the $8.90-\mathrm{MeV}$ was evaluated as around $10 \%$ of the total reaction rate. (c) is for the $9.20-\mathrm{MeV}$ resonance, where we also found a small peak in the spectrum. We used $J^{\pi}=5 / 2^{+}$ and the total width $\Gamma_{\text {tot }}=500 \mathrm{keV}$ from a previous measurement [17], although such a large $\Gamma_{\text {tot }}$ was inconsistent with our R-matrix analysis. (d) is for the $9.97-\mathrm{MeV}$ resonance, which was identified as a strong alpha resonance in the spectrum. The tentative assignment of $J^{\pi}=7 / 2^{-}$[17] was used for the evaluation, and a smaller contribution was obtained, as shown in Fig. 1. We also evaluated con- 
tributions for higher resonances, but none of them had an effect as much as or larger than the case (d).

In summary, the resonances at $8.90 \mathrm{MeV}$ and $9.20 \mathrm{MeV}$ have a possibility to give significant contributions to the reaction rate for $T_{9}=1.5-3$, although they are unlikely to be more than the contribution of the $8.420-\mathrm{MeV}$ resonance, which dominates the reaction rate. Considering that the $\Gamma_{\gamma}$ used here could be underestimated by factors and the decay widths and $J^{\pi}$ are also uncertain, more studies are necessary for the determination of resonant parameters in the excitation energy region of $E_{\text {ex }}=8.5-9.5 \mathrm{MeV}$, which might be difficult to access from the ${ }^{10} \mathrm{~B}+p$ channel. On the other hand, the resonances above 9.5 MeV can be considered as negligible for even higher temperature, $3<T_{9}<10$.

This work was partly supported by JSPS KAKENHI (No. 21340053,25800125) from the Ministry of Education, Culture, Sports, Science and Technology (MEXT) of Japan, and the Priority Centers Research Program in Korea (2009-0093817).

\section{References}

[1] S. Kubono, Y. Yanagisawa, T. Teranishi, S. Kato, T. Kishida, S. Michimasa, Y. Ohshiro, S. Shimoura, K. Ue, S. Watanabe et al., Eur. Phys. J. A13, 217 (2002)

[2] Y. Yanagisawa, S. Kubono, T. Teranishi, K. Ue, S. Michimasa, M. Notani, J.J. He, Y. Ohshiro, S. Shimoura, S. Watanabe et al., Nucl. Instrum. Meth. Phys. Res., Sect. A 539, 74 (2005)

[3] H. Yamaguchi, Y. Wakabayashi, G. Amadio, S. Hayakawa, H. Fujikawa, S. Kubono, J. He, A. Kim, D. Binh, Nucl. Instrum. Meth. Phys. Res., Sect. A 589, 150 (2008)

[4] K.P. Artemov, O.P. Belyanin, A.L. Vetoshkin, R. Wolskj, M.S. Golovkov, V.Z. Gol'dberg, M. Madeja, V.V. Pankratov, I.N. Serikov, V.A. Timofeev et al., Sov. J. Nucl. Phys 52, 408 (1990)

[5] T. Teranishi, S. Kubono, S. Shimoura, M. Notani, Y. Yanagisawa, S. Michimasa, K. Ue, H. Iwasaki, M. Kurokawa, Y. Satou et al., Phys. Lett. B 556, 27 (2003)

[6] J.J. He, S. Kubono, T. Teranishi, M. Notani, H. Baba, S. Nishimura, J.Y. Moon, M. Nishimura, H. Iwasaki, Y. Yanagisawa et al., Phys. Rev. C 76, 055802 (2007)

[7] H. Yamaguchi, Y. Wakabayashi, S. Kubono, G. Amadio, H. Fujikawa, T. Teranishi, A. Saito, J. He, S. Nishimura, Y. Togano et al., Phys. Lett. B 672, 230 (2009)

[8] J.J. He, S. Kubono, T. Teranishi, J. Hu, M. Notani, H. Baba, S. Nishimura, J.Y. Moon, M. Nishimura, H. Iwasaki et al., Phys. Rev. C 80, 015801 (2009)

[9] H. Yamaguchi, T. Hashimoto, S. Hayakawa, D.N. Binh, D. Kahl, S. Kubono, Y. Wakabayashi, T. Kawabata, T. Teranishi, Phys. Rev. C 83, 034306 (2011)

[10] M. Wiescher, J. Görres, S. Graff, L. Buchmann, F.K. Thielemann, Astrophys. J. 343, 352 (1989)

[11] C. Fröhlich, P. Hauser, M. Liebendörfer, G. Martínez-Pinedo, F.K. Thielemann, E. Bravo, N. Zinner, W. Hix, K. Langanke, A. Mezzacappa et al., Astrophys. J. 637, 415 (2006)

[12] S. Wanajo, H.T. Janka, S. Kubono, Astrophys. J. 729, 46 (2011)

[13] H. Yamaguchi, D. Kahl, Y. Wakabayashi, S. Kubono, T. Hashimoto, S. Hayakawa, T. Kawabata, N. Iwasa, T. Teranishi, Y. Kwon et al., Phys. Rev. C 87, 034303 (2013)

[14] M. Freer, N.L. Achouri, C. Angulo, N.I. Ashwood, D.W. Bardayan, S. Brown, W.N. Catford, K.A. Chipps, N. Curtis, P. Demaret et al., Phys. Rev. C 85, 014304 (2012)

[15] C. Angulo et al., Nucl. Phys. A 656, 3 (1999)

[16] Y. Xu, K. Takahashi, S. Goriely, M. Arnould, M. Ohta, H. Utsunomiya, Nucl. Phys. A 918, 61 (2013)

[17] M. Wiescher, R.N. Boyd, S.L. Blatt, L.J. Rybarcyk, J.A. Spizuoco, R.E. Azuma, E.T.H. Clifford, J.D. King, J. Görres, C. Rolfs et al., Phys. Rev. C 28, 1431 (1983) 